\title{
Cannabidiol (CBD) Consumption and Perceived Impact on Extrahepatic Symptoms in Patients with Autoimmune Hepatitis
}

\author{
Karan Mathur $^{1} \cdot$ Vahin Vuppalanchi $^{1} \cdot$ Kayla Gelow $^{1} \cdot$ Raj Vuppalanchi $^{1} \cdot$ Craig Lammert $^{1}$ (i)
}

Received: 24 May 2019 / Accepted: 19 July 2019 / Published online: 30 July 2019

(c) The Author(s) 2019

\begin{abstract}
Background and Aims Utilization and safety of cannabidiol (CBD) in patients with autoimmune hepatitis (AIH) are currently unknown. We aimed to identify the frequency of CBD use, impact on symptoms, and safety profile.

Methods An invitation to complete a CBD-specific questionnaire was posted every other day to well-established autoimmune hepatitis Facebook communities (combined membership of 2600 individuals) during a 10-day study period. Age $\geq 18$ years and an AIH diagnosis by a physician were the eligibility criteria for participation in the survey.

Results In total, $371 \mathrm{AIH}$ patients (median age 49 years, 32\% reported advanced fibrosis) completed the questionnaire. Respondents were 91\% women, 89\% Caucasian, and 89\% from North America. Ninety-three (25\%) respondents were ever CBD users, with 55 of them (15\% of the survey responders) identified as current users. Among ever users, $45.7 \%$ reported their treating doctors were aware of their CBD use. The most common reason cited for CBD use was pain (68\%), poor sleep (62\%), and fatigue (38\%). Most respondents using CBD for these symptoms reported a significant improvement in pain (82\%), sleep (87\%), and fatigue (61\%). In ever CBD users, $17.3 \%$ were able to stop a prescription medication because of CBD use: pain medication (47\%), immunosuppression (24\%), and sleep aids (12\%). Side effects attributed to CBD use were reported in $3 \%$ of CBD users, yet there were no reported emergency department visits or hospitalizations.

Conclusion CBD use was not uncommon in patients with $\mathrm{AIH}$, and its use was associated with reports of improvement in extrahepatic symptoms.
\end{abstract}

Keywords $\mathrm{AIH} \cdot \mathrm{CBD} \cdot$ Fatigue $\cdot$ Pain

\section{Introduction}

Autoimmune hepatitis (AIH) is a chronic liver disorder in which clinical presentation can vary widely from non-specific extrahepatic symptoms to fulminant hepatic failure [1]. Immunosuppressive therapy is the mainstay of treatment for AIH [2] and has been associated with improvement in symptoms, liver biochemistries, and histologic inflammation [3]. Unfortunately, impairment of emotional and mental health is prevalent in $\mathrm{AIH}$ and can diminish the health-related quality

Electronic supplementary material The online version of this article (https://doi.org/10.1007/s10620-019-05756-7) contains supplementary material, which is available to authorized users.

Craig Lammert

clammert@iu.edu

1 Division of Gastroenterology and Hepatology, Department of Medicine, Indiana University School of Medicine, 702 Rotary Circle, Suite 225, Indianapolis, IN 46202, USA of life in these patients $[4,5]$. The etiology of this phenomenon remains unclear, yet may be linked to common AIH symptoms such as pain, fatigue, and sleep disturbances [6, 7].

Complementary and alternative medical strategies have become a popular approach for patients with chronic diseases [8] and often are utilized to target pain, anxiety, and depression [9]. Cannabidiol (CBD), one of the more than 110 different cannabinoids that have been isolated by researchers from Cannabis Sativa plant, is more commonly known as marijuana. However, CBD does not cause intoxication or euphoria (the "high") that comes from tetrahydrocannabinol (THC), the psychoactive cannabinoid. CBD has received lot of recent attention after Food \& Drug Administration's (FDA) approval of purified CBD, Epidiolex, as treatment for two rare forms of epilepsy [10]. Utilization of CBD outside this indication for a variety of symptoms such as pain, glaucoma, anxiety has had mixed reviews and is not recognized by the FDA as an ailment 
that CBD can treat $[11,12]$. Furthermore, there is a shift in legal status after the 2018 Farm Bill allowing for expanded cultivation of industrial hemp and permission for the transfer of hemp-derived products across state lines for commercial or other purposes. Removal of hemp-derived products from Schedule 1 status has led to an explosion of CBD-related products to be available legally in some states. However, adding CBD, regardless of its extraction source, to food, beverages, and dietary supplements, is currently not permitted by the FDA.

Research in a rare disease is challenging due to limited access to patients. However, patients with rare disease are increasingly colocated virtually online through social media outlets such as Facebook, Instagram or through traditional Web sites that promote peer-to-peer connections (www.patientslikeme.com) or nonprofit organizations dedicated to their disease [13]. Our group in the past has successfully leveraged social media to conduct research in online cohorts of autoimmune hepatitis by deploying surveys that even included confirmation of diagnosis by procuring medical records and biosamples $[14,15]$. In the current study, we aimed to identify the frequency of CBD use in AIH patients and assess the impact on symptoms and safety profile using two large AIH-specific social media groups.

\section{Methods}

We conducted a cross-sectional survey study using AIH social media communities on Facebook (www.facebook. com): Autoimmune Hepatitis Research Network and Autoimmune Hepatitis Association. We have previously described this approach for collecting patient-reported disease attributes [16] as well as recruitment to an ongoing AIH biorepository at Indiana University [15]. At the time of the survey, the combined membership of these online communities was over 2600 . An online invitation to participate in a research study collecting information on CBD use was posted on these groups every other day for 10 days (October 2018). The survey included a link directed to an IRBapproved Redcap database for data collection.

\section{Survey Tool}

The questionnaire was composed of 33 questions (Supplementary Table 1) assessing participant demographics, AIH disease characteristics, history of and current CBD use, and adverse effects. Participants were required to be aged $\geq 18$ years and have previously received a diagnosis of AIH from a medical doctor.

\section{Statistical Analysis}

Survey data were analyzed using SPSS 25 software. Continuous variables were summarized as median and the 25 th and 75th percentiles, and $p$ values were obtained with the Wilcoxon rank-sum test.

\section{Results}

A total of 371 participants with AIH completed the questionnaire during the study period. The group was predominantly female (91.4\%) and Caucasian (88.9\%) and had a median age of 49 years at survey completion (Table 1). The median age of AIH diagnosis was 42 years. Of the 319 patients who reported having liver biopsy completed, $34.5 \%$ had early fibrosis, $32.1 \%$ had advanced fibrosis, and $34 \%$ did not know the stage of fibrosis reported on most recent biopsy.

\section{Immunosuppressant Therapy for AlH}

At the time of the survey, $88.1 \%$ of patients were on immunosuppressive medications: $35.2 \%$ on prednisone, $12.4 \%$ on budesonide, $52.3 \%$ on azathioprine (AZA), $6.5 \%$ on 6-mercaptopurine (6-MP), $13.2 \%$ on mycophenolate mofetil (MMF), $4.6 \%$ on tacrolimus, $0.8 \%$ on sirolimus, $1.1 \%$ on cyclosporine (CSA), and $12.4 \%$ on ursodeoxycholic acid (UDCA). In total, 198 participants (53.4\%) were on at least on one immunosuppressant, and 129 (34.8\%) were on more than one. There were 118 participants $(31.8 \%)$ on two immunosuppressants, 10 (2.7\%) on three, and $1(0.3 \%)$ on four. Among participants taking more than two immunosuppressants, 99 (76.7\%) were taking a combination of steroid and a thiopurine (AZA or 6-MP), 25 (19.3\%) a combination of steroid and MMF. Only 12 patients were taking more than one immunosuppressant beyond steroids: 4 (33.3\%) on tacrolimus and MMF, 4 (33.3\%) on tacrolimus and AZA, 1 (8.3\%) tacrolimus and AZA and sirolimus, 2 (16.7\%) CSA and AZA, and $1(8.3 \%)$ CSA and MMF.

There were 44 participants (11.9\%) not on immunosuppression at the time of the survey, of these, and 35 (79.5\%) had been treated previously with immunosuppression. Among all participants, former immunosuppression regimens had included prednisone $(224,60.4 \%)$, budesonide (56, 15.1\%), AZA (117, 31.5\%), 6-MP 14 (3.8\%), MMF $32(8.6 \%)$, tacrolimus $10(2.7 \%)$, sirolimus $2(0.5 \%)$, and CSA $9(2.4 \%)$. 
Table 1 Demographics and selected characteristics of AIH patients who participated in the current survey

\begin{tabular}{|c|c|}
\hline & AIH participants $(n=371)$ \\
\hline Age at survey completion (years) & $49(38,59)$ \\
\hline Age at AIH diagnosis (years) & $42(30,52.25)$ \\
\hline \multicolumn{2}{|l|}{ Race } \\
\hline Caucasian & $88.9 \%$ \\
\hline Hispanic & $3.2 \%$ \\
\hline Black & $2.2 \%$ \\
\hline Multiple & $2.4 \%$ \\
\hline Other & $2.4 \%$ \\
\hline Gender, $\%$ female & $91.4 \%$ \\
\hline \multicolumn{2}{|l|}{ Continent } \\
\hline North America & $81.4 \%$ \\
\hline Europe & $14.3 \%$ \\
\hline Australia & $2.2 \%$ \\
\hline Asia & $0.5 \%$ \\
\hline South America & $0.8 \%$ \\
\hline \multicolumn{2}{|l|}{ North America } \\
\hline USA & $76.8 \%$ \\
\hline Canada & $4.6 \%$ \\
\hline \multicolumn{2}{|l|}{ Gross household income/year } \\
\hline$<\$ 20,000$ & $9.4 \%$ \\
\hline$\$ 20,001-\$ 50,000$ & $27.0 \%$ \\
\hline$\$ 50,001-\$ 100,000$ & $32.6 \%$ \\
\hline$\$ 100,001-\$ 150,000$ & $18.6 \%$ \\
\hline$>\$ 150,000$ & $11.4 \%$ \\
\hline \multicolumn{2}{|l|}{ Level of education } \\
\hline GED & $13.7 \%$ \\
\hline Bachelor's degree & $24.5 \%$ \\
\hline Graduate degree & $19.0 \%$ \\
\hline Some college & $32.1 \%$ \\
\hline Technical school & $8.1 \%$ \\
\hline Some high school & $1.6 \%$ \\
\hline \multicolumn{2}{|l|}{ Fibrosis on biopsy } \\
\hline $\mathrm{F} 1$ & $18.9 \%$ \\
\hline $\mathrm{F} 2$ & $15.8 \%$ \\
\hline $\mathrm{F} 3$ & $19.7 \%$ \\
\hline $\mathrm{F} 4$ & $12.8 \%$ \\
\hline Don’t know & $28.6 \%$ \\
\hline No biopsy completed & $3.5 \%$ \\
\hline \multicolumn{2}{|l|}{ Current medication } \\
\hline Prednisone & $35.3 \%$ \\
\hline Budesonide & $12.4 \%$ \\
\hline 6-Mercaptopurine & $6.5 \%$ \\
\hline Azathioprine & $52.3 \%$ \\
\hline Mycophenolate mofetil & $13.2 \%$ \\
\hline Tacrolimus & $4.6 \%$ \\
\hline Cyclosporine & $1.1 \%$ \\
\hline Sirolimus & $0.8 \%$ \\
\hline Ursodeoxycholic acid & $12.4 \%$ \\
\hline None & $11.9 \%$ \\
\hline
\end{tabular}

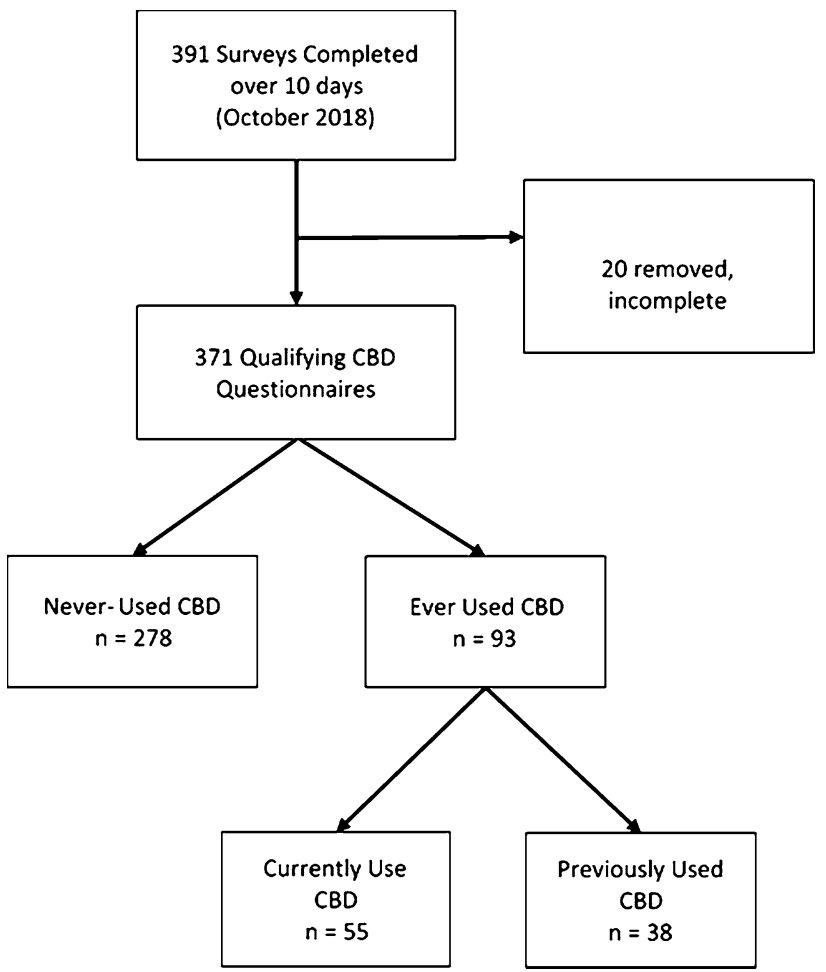

Fig. 1 Survey completion and CBD use among AIH patients

\section{CBD Therapy}

In total, $93(25.1 \%)$ patients reported they had ever used CBD for the treatment of AIH or its perceived symptoms (Fig. 1). Ever CBD users were similar to never CBD users; however, ever users tended to be female $(p<0.09)$ and have lower household incomes $(p<0.07)$ (Table 2). Ever CBD users were also more likely not to be taking any other therapy for the treatment of AIH (12.9\% vs. 6\%, $p=0.035)$. In total, $278(74.9 \%)$ participants reported they never used CBD because of high cost $(18.3 \%)$ or they never heard of it (17.6\%), but also worried about positive drug screening (10.4\%), local laws (9.7\%), side effects $(4.7 \%)$, effectiveness (3.2\%), and moral or religious issues (3.2\%). Knowledge about CBD use was attributed to information from social media $(78.5 \%)$, family/friends (69.9\%), other patients (24.7\%), doctors (20.4\%), and other media (19.4\%).

Among ever CBD users, 55 (59\%) were current users for the treatment of AIH and 38 (41\%) were prior users. Among ever users, $45.7 \%$ reported their treating doctors were aware of their CBD use at the time of use. The most frequent AIH symptoms treated by participants with CBD were poor sleep (66.7\%), pain (66.7\%), fatigue (44.1\%), "liver inflammation" (29\%), and itch (10.8\%). Survey participants reported CBD used resulted in significant improvement in sleep (54/59, $93 \%)$, pain $(55 / 63,87.3 \%)$, fatigue $(25 / 38,65.8 \%)$, "liver 
Table 2 Demographics and selected characteristics of AIH patients according to $\mathrm{CBD}$ ever versus never use

\begin{tabular}{|c|c|c|c|}
\hline & CBD ever & CBD never & $p$ \\
\hline Age at survey completion, median (Q1, Q3) & $47(37.5,58)$ & $50(38,60)$ & 0.21 \\
\hline Age at AIH diagnosis, median (Q1, Q3) & $42(30,50)$ & $42(30,53)$ & 0.78 \\
\hline \multicolumn{4}{|l|}{ Race } \\
\hline Caucasian & $87.10 \%$ & $89.90 \%$ & \multirow[t]{3}{*}{0.66} \\
\hline Hispanic & $2.20 \%$ & $3.60 \%$ & \\
\hline Black & $2.20 \%$ & $2.20 \%$ & \\
\hline \multicolumn{4}{|l|}{ Gender } \\
\hline Female & $95.70 \%$ & $90 \%$ & \multirow[t]{2}{*}{0.09} \\
\hline Male & $4.30 \%$ & $10 \%$ & \\
\hline \multicolumn{4}{|l|}{ Continent } \\
\hline North America & $81 \%$ & $83.50 \%$ & \multirow[t]{4}{*}{0.45} \\
\hline Europe & $18.30 \%$ & $13.10 \%$ & \\
\hline Australia & $1.10 \%$ & $2.60 \%$ & \\
\hline South America & $0.00 \%$ & $1.10 \%$ & \\
\hline \multicolumn{4}{|l|}{ North America } \\
\hline USA & $77.40 \%$ & $76.60 \%$ & \multirow[t]{2}{*}{0.76} \\
\hline Canada & $3.20 \%$ & $5 \%$ & \\
\hline \multicolumn{4}{|l|}{ Gross household income/year } \\
\hline$<\$ 20,000$ & $9.70 \%$ & $9.90 \%$ & \multirow[t]{5}{*}{0.07} \\
\hline$\$ 20,001-\$ 50,000$ & $36.50 \%$ & $24.50 \%$ & \\
\hline$\$ 50,001-\$ 100,000$ & $36.50 \%$ & $32.10 \%$ & \\
\hline$\$ 100,001-\$ 150,000$ & $3.33 \%$ & $21.50 \%$ & \\
\hline$>\$ 150,000$ & $8.60 \%$ & $12.40 \%$ & \\
\hline \multicolumn{4}{|l|}{ Level of education } \\
\hline GED & $11.80 \%$ & $14.40 \%$ & \multirow[t]{6}{*}{0.31} \\
\hline Bachelor's degree & $21.50 \%$ & $25.50 \%$ & \\
\hline Graduate degree & $14 \%$ & $21.20 \%$ & \\
\hline Some college & $40.10 \%$ & $29.60 \%$ & \\
\hline Technical school & $9.70 \%$ & $7.70 \%$ & \\
\hline Some high school & $2.20 \%$ & $1.50 \%$ & \\
\hline \multicolumn{4}{|l|}{ Fibrosis on biopsy } \\
\hline $\mathrm{F} 1$ & $18.30 \%$ & $19.40 \%$ & \multirow[t]{4}{*}{0.38} \\
\hline $\mathrm{F} 2$ & $19.40 \%$ & $14.70 \%$ & \\
\hline F3 & $24.70 \%$ & $18 \%$ & \\
\hline F4 & $11.80 \%$ & $13.20 \%$ & \\
\hline \multicolumn{4}{|l|}{ Current medical treatment for AIH } \\
\hline Prednisone & $39.80 \%$ & $33.80 \%$ & 0.3 \\
\hline Budesonide & $12.90 \%$ & $12.20 \%$ & 0.87 \\
\hline 6-Mercaptopurine & $7.50 \%$ & $6.10 \%$ & 0.63 \\
\hline Azathioprine & $41.90 \%$ & $55.80 \%$ & 0.02 \\
\hline Mycophenolate mofetil & $14.00 \%$ & $12.90 \%$ & 0.8 \\
\hline Tacrolimus & $4 \%$ & $5.40 \%$ & 0.67 \\
\hline None & $12.90 \%$ & $6.10 \%$ & 0.035 \\
\hline
\end{tabular}

inflammation" (27/32, 84.4\%), and itch (7/8, 87.5\%). Seventeen (18.3\%) ever CBD users reported they were able to stop or reduce a prescription medication because of CBD use. Drug classes reduced or stopped included pain (11/17; 5 narcotics, 4 NSAIDs, two other), immunosuppressants (4/17; 3 steroids, 1 AZA), anxiolytics (3/17; 2 lorazepam, one diazepam), antidepressants (1/17; sertraline), and sedatives (1/17; zolpidem).

CBD was administered most frequently via sublingual drops $(55.9 \%)$, oral capsule (32.3\%), inhalation (28\%), and topically $(12.9 \%)$. Only a minority of participants reported characteristics of CBD dose (29\%). Among 
reported dosing information, the median dose was $20 \mathrm{mg}$ ( $5 \mathrm{mg}, 200 \mathrm{mg}$ ), the median daily frequency was 1 (1, 2), and total daily dose was $25.5 \mathrm{mg}(4.8 \mathrm{mg}, 87.5 \mathrm{mg}$ ). The median duration of CBD use among current CBD users was 3.5 months $(1,6)$ and was significantly more than prior CBD users ( 1 month $(1,3), p=0.007)$. The most common cause for stopping CBD was cost (50\%), worry about positive drug test $(10.5 \%)$, side effects $(10.5 \%)$, ineffective $(7.9 \%)$, the doctor advised against CBD (7.9\%), and change in the law (7.1\%). There was no difference in the various demographics and medication usage between ever CBD users in either of the histology groups (Table 3 ).

Serious side effects attributed to CBD therapy were reported by three participants $(3.2 \%)$ and included: hunger (1), dry mouth (1), red eyes (1), euphoria (1), and itchiness (1). Two participants reported side effects were significant enough to seek their doctor's advice, but there were no emergency room visits or hospitalizations reported.

\section{Discussion}

CBD has been used to treat various symptoms in the gastroenterology field with variable outcomes [17-20], yet its use among patients with chronic liver disease remains unknown [21]. In the current study, we observed that $25 \%$ of study participants reported ever CBD use directed at the most frequent AIH-related extrahepatic symptoms: pain, sleep, and fatigue. Females and those with lower household incomes were more likely to have used CBD, yet many patients reported their treating doctor was unaware of their use. A majority of ever CBD users saw significant improvement in targeted symptoms with CBD use and very few experienced severe side effects.

The FDA, when supported by rigorous scientific research, has endorsed medical use of marijuana-derived products as drugs for the treatment of disease. Extrapolating the approval of a purified CBD derived from marijuana for a seizure disorder to hemp-derived use of CBD to treat a variety of symptoms can be serious, unproven, risky, and dangerous. Furthermore, in the Epidiolex-treated patients in

Table 3 Demographics and selected characteristics of AIH patients stratified by fibrosis according to CBD ever versus never use

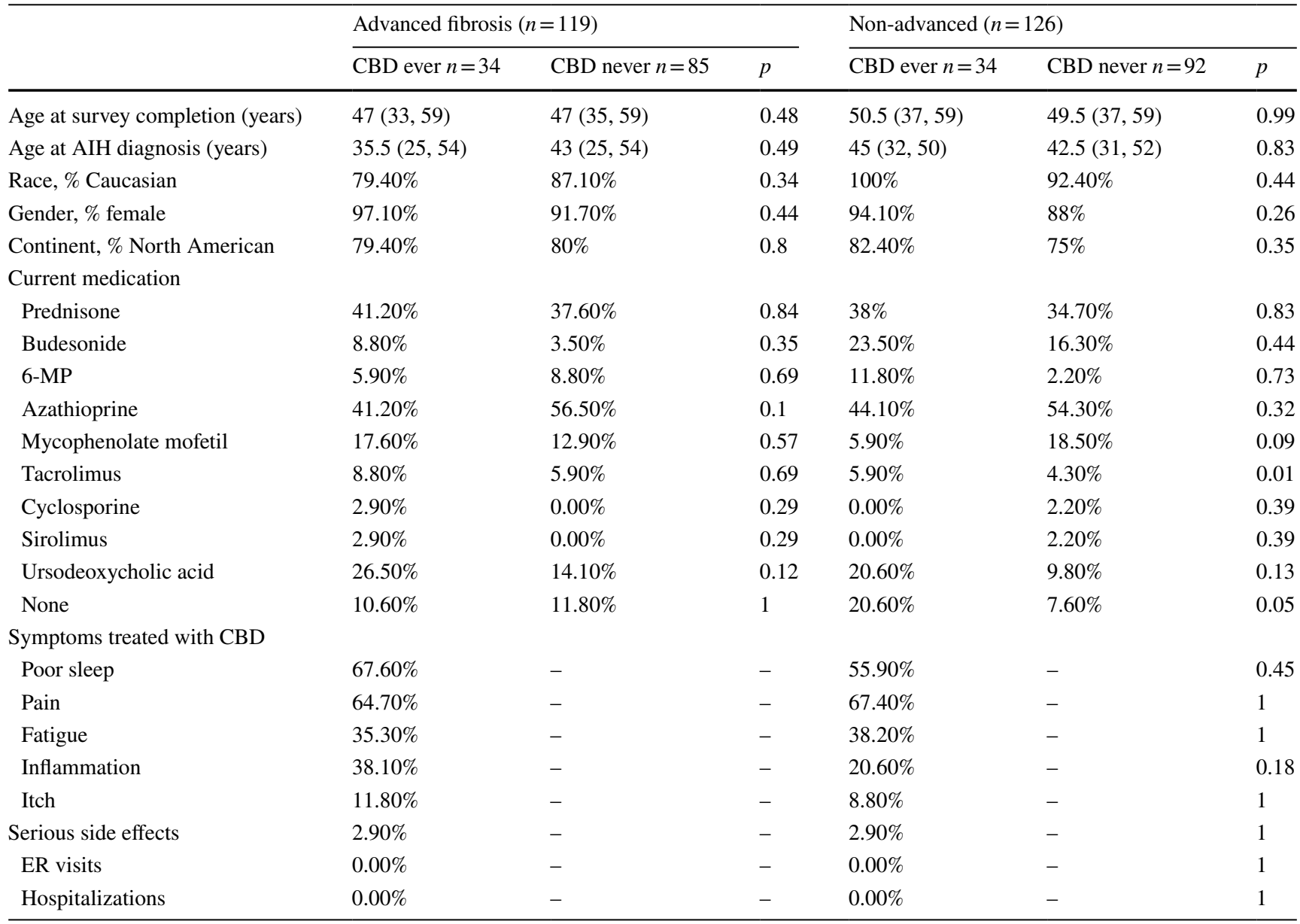


the clinical trials, sleepiness, sedation, and lethargy; elevated liver enzymes; decreased appetite; diarrhea; rash; fatigue, malaise and weakness, insomnia, sleep disorder, and poor quality sleep; and infections were reported. Therefore, the CBD use among the patients with AIH as evidenced by the survey respondents is concerning. The usage varied equally among the age groups and liver fibrosis staging suggesting no rationale or vulnerability of specific demographics or disease phenotype from illegal marketing. Depending on the percentage of THC in a product and frequency of use, a positive urine drug screen for cannabinoids is possible. This observation could pose a significant hindrance for patients undergoing liver transplant evaluation for end-stage liver disease associated with AIH.

CBD, a potent inhibitor of CYP3A4 and CYP2C19, can target a variety of channels and receptors including human TRP and voltage-gated channels, serotonin receptors 5-HT1 A and 5-HT2A, and G-coupled protein and adenosine receptors [22]. Data on drug interactions in human subjects taking CBD are scarce; however, we hypothesize that there is risk of altered pharmacokinetics in CBD or concurrently administered drugs undergoing metabolism via these CYP systems. The steroid drug class and calcineurin inhibitors [30], metabolized via CYP3A4, are of significant concern given 177 patients $(47.7 \%)$ of study respondents reported current use of either prednisone or budesonide and 21 patients $(5.7 \%)$ reported use of either tacrolimus or cyclosporine. The relative infrequency that patients did not report CBD use to their treating doctors $(45.7 \%)$ and product variability across manufacturers [23, 24], dose [21], and route [25] of administration could further expand this risk.

No published data encapsulate CBD use across populations, yet the frequency of CBD use reported in our study is similar to that observed among adults with liver disease in the USA using other complementary and alternative medicines [26]. Health-related quality of life in AIH is significantly reduced $[27,28]$; thus, the use of CBD by patients to mitigate symptoms attributed to AIH outside the liver is not surprising. A majority of patients reported significant improvement in AIH-related indications for use (pain, poor sleep, fatigue, and itch) which included very few serious side effects. Other data about the improvement in these symptoms and safety profiles within other CBD-treated populations are unavailable, but our observations are congruent with a meta-analysis that assessed all cannabinoids for medical use [20].

We readily admit the possible limitation of patientreported disease and CBD use data in this study. Historically, we have shown that AIH patients participating in similar research approaches exhibit excellent diagnosis agreement when compared to their medical records [15] as well as similar demographics and disease attributes [16]. However, we also believe there are tremendous benefits to treating physicians and patients allowed by rapid recruitment and dissemination of research regarding emerging and popular healthcare trends [29]. Furthermore, the anonymity offered through social media may allow for unbiased answers for a sensitive topic such as CBD use. CBD has been recently showing up in everything from beauty creams and bath bombs to vape pens, seltzer, and snacks; thus, our survey may underestimate total CBD use among patients. Furthermore, attribution of all reported extrahepatic symptom improvement to CBD use in our study is inappropriate, as we did not collect other interventions or exposures (marijuana, other over the counter supplements, meditation, etc.) that may have been completed concurrently with CBD.

In summary, CBD use in our online cohort was common and associated with a significant improvement in AIHrelated symptoms and side effects of immunosuppressant medications. The overall positive therapeutic benefits from this study as well as the observation of few serious side effects would be supportive of future trials (possibly with cannabinoids) aimed at improving health-related quality of life in $\mathrm{AIH}$ patients. Strict regulation of product formulation and dose and well as further examination of CBD pharmacokinetics would help alleviate safety concerns in patients taking a wide variety of pharmaceuticals.

Funding Funding was provided by National Institute of Diabetes and Digestive and Kidney Diseases (Grant No. DK114561).

\section{Compliance with Ethical Standards}

Conflict of interest The authors declare that they have no conflict of interest.

Open Access This article is distributed under the terms of the Creative Commons Attribution-NonCommercial 4.0 International License (http://creativecommons.org/licenses/by-nc/4.0/), which permits any noncommercial use, distribution, and reproduction in any medium, provided you give appropriate credit to the original author(s) and the source, provide a link to the Creative Commons license, and indicate if changes were made.

\section{References}

1. Heneghan MA, Yeoman AD, Verma S, et al. Autoimmune hepatitis. Lancet. 2013;382:1433-1444.

2. EASL Clinical Practice Guidelines. Autoimmune hepatitis. $J$ Hepatol. 2015;63:971-1004.

3. Manns MP, Czaja AJ, Gorham JD, et al. Diagnosis and management of autoimmune hepatitis. Hepatology. 2010;51:2193-2213.

4. Takahashi A, Moriya K, Ohira H, et al. Health-related quality of life in patients with autoimmune hepatitis: a questionnaire survey. PLoS ONE. 2018;13:e0204772.

5. Vuppalanchi V, Chalasani S, Mohammad S, et al. Patient reported outcomes of physical and psychological suffering in patients with 
autoimmune hepatitis. In: American College of Gastroenterology. October 5-10, 2019, Philadelphia. Abstract \#P2376.

6. Janik MK, Wunsch E, Raszeja-Wyszomirska J, et al. Autoimmune hepatitis exerts a profound, negative effect on healthrelated quality of life: a prospective, single-centre study. Liver Int. 2019;39:215-221.

7. van der Plas SM, Hansen BE, de Boer JB, et al. Generic and disease-specific health related quality of life of liver patients with various aetiologies: a survey. Qual Life Res. 2007;16:375-388.

8. Bishop FL, Lewith GT. Who uses CAM? A narrative review of demographic characteristics and health factors associated with CAM use. Evid Based Complement Altern Med eCAM. 2010;7:11-28.

9. Barnes PM, Powell-Griner E, McFann K, et al. Complementary and alternative medicine use among adults: United States. $A d v$ Data. 2002;2004:1-19.

10. O'Connell BK, Gloss D, Devinsky O. Cannabinoids in treatmentresistant epilepsy: a review. Epilepsy Behav. 2017;70:341-348.

11. Hasenoehrl C, Storr M, Schicho R. Cannabinoids for treating inflammatory bowel diseases: Where are we and where do we go? Expert Rev Gastroenterol Hepatol. 2017;11:329-337.

12. Kafil TS, Nguyen TM, MacDonald JK, et al. Cannabis for the treatment of Crohn's disease. Cochrane Database Syst Rev. 2018;11:Cd012853.

13. Schumacher KR, Stringer KA, Donohue JE, et al. Social media methods for studying rare diseases. Pediatrics. 2014;133:e1345-e1353.

14. Chalasani S, Vuppalanchi V, Tilmans L, et al. Novel approach leveraging social media indicates complementary and alternative medicine use highly prevalent and is sometimes associated with serious adverse events in patients with autoimmune hepatitis. American Journal of Gastroenterology. 2018;113:S526.

15. Comerford M, Fogel R, Bailey JR, et al. Leveraging social networking sites for an autoimmune hepatitis genetic repository: pilot study to evaluate feasibility. J Med Internet Res. 2018;20:e14.

16. Fogel R, Comerford M, Chilukuri P, et al. Extrahepatic autoimmune diseases are prevalent in autoimmune hepatitis patients and their first-degree relatives: survey study. Interact J Med Res. 2018;7:e18.

17. Izzo AA, Camilleri M. Emerging role of cannabinoids in gastrointestinal and liver diseases: basic and clinical aspects. Gut. 2008;57:1140-1155.

18. Naftali T, Lev LB, Yablecovitch D, et al. Treatment of Crohn's disease with cannabis: an observational study. Isr Med Assoc J. 2011;13:455-458.
19. Naftali T, Bar-Lev Schleider L, Dotan I, et al. Cannabis induces a clinical response in patients with Crohn's disease: a prospective placebo-controlled study. Clin Gastroenterol Hepatol. 2013;11(1276-1280):e1.

20. Whiting PF, Wolff RF, Deshpande S, et al. Cannabinoids for medical use: a systematic review and meta-analysis. JAMA. 2015;313:2456-2473.

21. Goyal H, Rahman MR, Perisetti A, et al. Cannabis in liver disorders: a friend or a foe? Eur J Gastroenterol Hepatol. 2018;30:1283-1290.

22. Rong C, Carmona NE, Lee YL, et al. Drug-drug interactions as a result of co-administering delta(9)-THC and CBD with other psychotropic agents. Expert Opin Drug Saf. 2018;17:51-54.

23. Peace MR, Butler KE, Wolf CE, et al. Evaluation of two commercially available cannabidiol formulations for use in electronic cigarettes. Front Pharmacol. 2016;7:279.

24. Bonn-Miller MO, Loflin MJE, Thomas BF, et al. Labeling accuracy of cannabidiol extracts sold online. JAMA. 2017;318:1708-1709.

25. Huestis MA. Human cannabinoid pharmacokinetics. Chem Biodivers. 2007;4:1770-1804.

26. Henson JB, Brown CL, Chow SC, et al. Complementary and alternative medicine use in United States adults with liver disease. $J$ Clin Gastroenterol. 2017;51:564-570.

27. Wong LL, Fisher HF, Stocken DD, et al. The impact of autoimmune hepatitis and its treatment on health utility. Hepatology. 2018;68:1487-1497.

28. Schramm C, Wahl I, Weiler-Normann C, et al. Health-related quality of life, depression, and anxiety in patients with autoimmune hepatitis. J Hepatol. 2014;60:618-624.

29. Kulanthaivel A, Fogel R, Jones J, et al. Digital cohorts within the social mediome: an approach to circumvent conventional research challenges? Clin Gastroenterol Hepatol. 2017;15:614-618.

30. Leino AD, Emoto C, Fukuda T, Privitera M, Vinks AA, Alloway RR. Evidence of a clinically significant drug-drug interaction between cannabidiol and tacrolimus. Am J Transplant. 2019. https ://doi.org/10.1111/ajt.15398.

Publisher's Note Springer Nature remains neutral with regard to jurisdictional claims in published maps and institutional affiliations. 